\title{
Coordination and artifacts in joint activity: the case of tagging in high-risk industries
}

\author{
François Palaci ${ }^{\mathrm{a}, \mathrm{b}, *}$, Geneviève Filippi ${ }^{\mathrm{a}}$ and Pascal Salembier ${ }^{\mathrm{b}}$ \\ ${ }^{a}$ Groupe Facteurs Humains, Département Management des Risques Industriels, EDF R\&D, 1 avenue du Général \\ de Gaulle, 92140 Clamart, France \\ ${ }^{\mathrm{b}}$ Équipe Tech-CICO, Institut Charles Delaunay, Université de Technologie de Troyes, 12 rue Marie Curie, 10010 \\ Troyes, France
}

\begin{abstract}
The social, organizational, and technological complexity of high-risk organizations creates a strong need for coordination. Joint activity in such systems thus relies both on formal coordination and on a set of informal coordinative practices. Coordinated joint activity is documented in this paper from two perspectives: the development of coordinative practices to overcome the limitations of formal coordination, and the use of artifacts for coordinative purposes. The empirical material is provided by a workplace study undertaken according to theoretical conceptions of situated action and cognition. It was undertaken in the context of a design project that aims at improving the tagout process in a high-risk industry. Findings first describe and analyze situations in which formal coordination turned out to be more of a constraint than a resource for effective action. They then illustrate the role played by artifacts in coordinated activity, focusing on the use of a particular artifact, the tagout tag, in different situations.
\end{abstract}

Keywords: coordination, team work, artifact, design, nuclear.

\section{Introduction}

Over recent years, the Nuclear Power Generation Division of EDF (Electricity of France) has been implementing numerous innovation projects that aim at improving plant outages performance regarding duration as well as personnel security and nuclear safety. The main drivers for these projects are to cope with a significant increase in volumes of maintenance activities expected in the short term, and to benefit from the possibilities offered by modern ICT systems to simplify requirements for workers standards and procedures. Specifically, improving the efficiency of the tagout process (which purpose is to secure maintenance activities) is considered as a high-stake objective.

Within this context, the question arose to determine whether North American work organization regarding tagouts and its supporting tools could be of interest for redesigning EDF ones, and if so, to what extent. The implicit assumption is that introducing new technologies or new organizational settings will automatically enhance productivity and safety. Notwithstanding, the idea that innovation should be informed by workplace studies is beginning to spread among managers. Indeed, there is a recognition that failures of innovative devices often derive from a poor understanding of actual work practices.

Thus, a research project in ergonomics is currently undertaken with the objective of identifying and understanding cultural, organizational, and human success factors of the tagout process in EDF nuclear power plants and in the US, in order to contribute to the redesign of the EDF one. This paper presents the first step of this research project dealing with current tagout practices in EDF plants.

\section{The tagout process as a joint activity}

The tagout process is highly distributed within space, time, and between practitioners. It is also mediated by many artifacts. Its underlying social, organizational, and technological complexity creates a

\footnotetext{
Corresponding author. E-mail: francois@palaci.fr.
} 
strong need for coordination, for 'managing dependencies between activities' [9]. The tagout process can thus be thought of as the manifestation of a joint activity, 'one that is carried out by an ensemble of people acting in coordination with each other' [4]. A crucial question to address when redesigning the tagout process is then to understand how coordination is achieved in this joint activity and when, why, how, it breaks up.

Previous research (e.g., [7]) has mentioned two complementary ways to achieve a coordinated joint activity:

- By means of formal coordination, that is to say pre-defined procedures or plans that, when objectified in artifacts, constitute what Schmidt and Simone term 'coordination mechanisms' [14];

- By means of ad hoc coordinative practices closely related to phenomena such as 'mutual awareness' [12], 'shared situation awareness' [3], 'common ground' [8].

The latter have been investigated in a variety of high-risk sectors: subway traffic management [6], emergency calls management [2], air traffic control [11], nuclear power plant control in accidental situations [15], hospitals [1]. Yet, this extensive body of research focused on real-time distributed collective activity, and little is known about coordinative practices of workers who are distributed during a longer time span [10], as in the case of practitioners involved in the tagout process working on different shifts. Now regarding 'coordination mechanisms', the 'need to investigate - thoroughly, systematically, and critically - their actual use' [13] still stands true.

\section{Tagging in EDF nuclear power plants}

Every time a maintenance activity has to be performed on an equipment, this equipment has to be isolated and appropriate safety conditions provided to workers. The purpose of the tagout process is both to isolate equipments requiring maintenance and to protect personnel from injury by controlling hazards.

Before proceeding with the working stage, the Maintenance department must appoint a work manager. Next, it requires a tagout from the tagout supervisor (Operations department) to get the equipment to be worked on isolated.

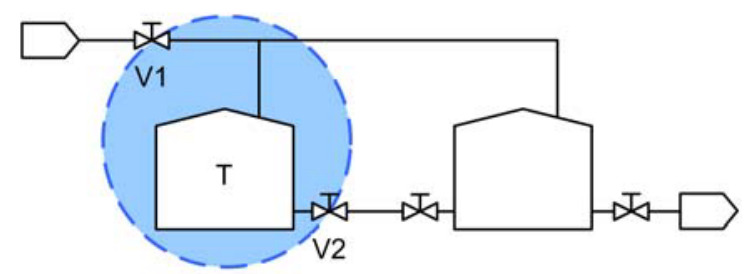

Fig. 1: A simplified example of an isolation boundary: to secure a maintenance activity on tank $\mathrm{T}$, the system would be drained and valves V1 and V2 closed and locked.

The tagout request is then reviewed twice, both by an off-shift tagout supervisor and an on-shift tagout supervisor. Once the shift manager has approved the tagout and the Maintenance department has confirmed the activity can take place, the on-shift tagout supervisor edits a tag hang sheet and gives it to a field operator. The energy isolating devices (e.g., breakers or valves) mentioned on the sheet are then placed in the required position (e.g., opened, closed or fused removed) and padlocked by the field operator. Eventually, a tag is attached on each locked device by the field operator, which explicitly mentions that the device must not be manipulated. A boundary within which no hazardous energy remains is thus formed (Figure 1 provides a simplified example).

The tagout supervisor can then issue the tagout certificate to the work manager, which assures her/him the isolation boundary is formed. Once the activity is over, the tagout certificate is given back to the tagout supervisor, tagout is removed and equipments are returned to service.

\section{Methods}

Data collection and analysis were undertaken according to theoretical assumptions drawn from situated action and cognition.

\subsection{Empirical data collection}

Data was collected in an ethnographic way. Data collection spread out over six shifts (i.e. 48 hours) during which four tagout supervisors were observed throughout their activity in an EDF nuclear power plant made up of two production units (i.e. two reactors). Field notes of the tagout supervisor's actions and verbal interactions were made. They were complemented with audio recordings of their verbal in- 
teractions, of handovers and of shift briefings. The different media supporting the observed activity (shift log, consulted documents and procedures) were systematically photographed.

To gain a better insight of the meaning, from the actor's point of view, of the observed activity, retrospective interviews were carried out: when the tagout supervisor seemed available to the researcher, she/he was asked questions about what happened just before so that she/he might explain her/his actions, verbal interactions and reasoning, and give her/his interpretation of previous events. For instance: 'A moment ago, when you saw the tag, you said that you did not understand. What was it?'.

Field notes and recordings have been transcribed.

\subsection{Data analysis}

\subsubsection{Building activity logs}

This step consists in copying in the two opposite columns of a table (see Table 1 for an example):

- An account of activity on the basis both of the researcher's observations and of external factors, e.g. aspects of the context such as the state of the nuclear process (first column). It is made from the transcripts of field notes and verbal interactions recordings, and the photographs

- The actor's point of view on her/his own activity (second column). It is built from the transcripts of retrospective interviews.

\subsubsection{Dividing activity logs and building cases}

This step consists in dividing activity logs into sequences of action. The move from one sequence to the other is decided when there is a change in the tagout supervisor's concerns in relation with her/his dynamic situation (e.g., in the excerpt provided in Table 1, the concern of the tagout supervisor is to understand why the tag is stapled to the tagout certificate).
Table 1: Excerpt from an activity $\log$.

\begin{tabular}{ll}
\hline $\begin{array}{l}\text { Actor's actions and verbal } \\
\text { interactions }\end{array}$ & $\begin{array}{l}\text { Actor's comments on his ac- } \\
\text { tions and verbal interactions }\end{array}$ \\
\hline The maintenance worker tells & 'The tag, it was stapled, so... I \\
the tagout supervisor: 'There is & $\begin{array}{l}\text { guess, by the tagout supervisor } \\
\text { who issued the tagout certifi- } \\
\text { a tag stapled to the tagout }\end{array}$ \\
certificate.' & $\begin{array}{l}\text { cate. Since on a device... that } \\
\text { is my interpretation, there was }\end{array}$ \\
The tagout supervisor says: & an OT, an OT that was lock- \\
'Why is there a tag stapled & ing... He removed the tag and \\
there? What is this again?' & he put it back...'
\end{tabular}

Cases are built by identifying continuous sequences of action that proceed from a single intrinsic set of themes. For instance, while a tagout supervisor was trying to understand the reason why a tag was stapled to the certificate (concern 1), he was interrupted by a phone call (concern 2). When the call was over, he tried again to understand why the tag was stapled to the certificate (back to concern 1). A case entitled 'the stapled tag' is thus built.

\subsubsection{Analyzing cases}

Ten cases that include empirical phenomena dealing with coordinative practices or the use of artifacts for coordinative purposes were selected to be analyzed. In each selected case, the tagout supervisor's continuous course of action was analyzed by attempting to document:

- Her/his concerns in relation with her/his dynamic situation;

- The elements of the setting that are meaningful to her/him;

- Her/his expectations about upcoming events;

- The knowledge, built upon her/his experience and professional culture, she/he makes use of.

\section{Findings}

Findings put into evidence two issues. The first one, presented in the next subsection, is that a coordinated joint activity cannot be achieved by formal coordination alone.

\subsection{Ad hoc practices to overcome the limitations of formal coordination}

In order to manage dependencies (whether human or technical) in the tagout process, formal coordination exists, that often takes the form of written procedures or computerized forms. In this section, cases (cf. 'Methods' section) are reported in which formal 
coordination turned out to be more of a constraint than a resource for effective action. In these cases, practitioners achieved efficiency nonetheless.

\subsubsection{Collective sense making to handle procedures that lack clarity}

Before some equipment can be returned to service after a maintenance activity, tests are required. Those tests are meant to check if the equipment itself is working and if it fulfils its function within the broader system it is included in. Such tests are usually carried out by practitioners from the same department in accordance with specified procedures.

In the situation reported here, though, the affected equipment was in the control room and, as a consequence, had to be tested both by workers from the Automatism department and by control room operators (Operations department). Yet, the main test procedure was referring only to a procedure from the Automatism department. The question then arose to determine who had to do what among practitioners from the two departments.

This lead to a long exchange between no less than five people (two automatism specialists, a control room operator, the assistant shift manager and the tagout supervisor). They eventually noticed a step in the procedure from the Automatism department that implied actions that can only be taken by the control room operators. They understood that the procedure corresponding to these actions was not explicitly mentioned because it did not exist for the precise affected equipment. The tagout supervisor then created the necessary procedure by copying the corresponding one from the second production unit.

Practitioners involved in this situation know they are in a situation (testing a control room equipment) in which they need to observe formal coordination. The procedure they are provided with being unclear, they engage in a collective sense making activity. Once they have made sense of the situation, the tagout supervisor acts so that the equipment can be returned to service without delay.

\subsubsection{Dealing with situations in which computerized procedures are inadequate}

When they send a tagout request to the tagout supervisor, practitioners from the Maintenance department need to fill a computerized form by providing, among other pieces of information, a title that summarize the activity, the serial numbers of the equipment to be worked, and the serial numbers of energy isolating devices to be padlocked prior to work.
In the situation reported here, the tagout supervisor received a request in which the title and the mention of the equipment to be worked were not coherent. The title mentioned a duckboards floor while the equipment was a pump. Furthermore, no energy isolating device was mentioned; instead, a remark was attached that said 'energy isolating devices are voluntarily not mentioned'.

The tagout supervisor gave a phone call to the Maintenance department to figure out what was the intended activity. He could have simply rejected the request but explained during the retrospective interview that his job was 'facilitating the realization of maintenance activities schedule'.

The Maintenance professional told the tagout supervisor that the tagout request he made was about the duckboards floor, which is located below the mentioned pump. He explained that this floor has no serial number, which makes it impossible to designate in the tagout request computerized form; he mentioned the pump instead.

The tagout supervisor discussed the matter with the assistant shift manager and they came to the conclusion that the activity could be carried out with a no-tagout work authorization.

In this situation, like in the one reported in the previous section, a collective sense making activity takes place. Its roots are of a different nature, though; they are to be found in a 'coordination mechanism' (the tagout request computerized form) that does not allow for a particular class of situations to be handled (equipments without serial number to be worked). Like in the previous situation, the outcome is efficient and, interestingly, it does not consist in circumventing formal coordination but in thinking of another formal way to deal with the issue at hand. But what if the situation had been such that the use of a no-tagout work authorization had not been an option?

\subsubsection{Cumbersome formal coordination bypassed by efficient practices}

In the situation reported here, an activity takes place in the radioactive area of the plant, and a watertightness test on the equipment being repaired is planned in the end of the activity. Yet, the isolation boundary securing the activity includes the water arrival that was closed and locked by Operations.

In strict accordance with formal coordination, once the activity was completed, the maintenance worker should have gone out of the radioactive area, given the tagout certificate back to the tagout supervisor, waited for a field operator from Operations to unlock 
and reopen the water arrival, gone back to the radioactive area to perform the test. In the event of a leakage during the test, he should have gone back and request a new tagout, ad libitum...

The actual practice is different. A field operator from Operations accompanies the maintenance worker. That way, when the activity is over, the field operator re-opens the water arrival and the test is performed. If the equipment is leaking, the field operator can close the water arrival and the maintenance worker adjust the repair. The tagout certificate is given back when both the activity and the test are done.

This situation provides a good example of a coordinative practice that is used by practitioners to bypass the cumbersome procedure of formal coordination and achieve efficiency. It is important to stress that safety is not forgotten in this widely used practice since the field operator remains beside the maintenance worker all the time.

\subsection{Artifacts used for coordinative purposes: the example of tagout tags}

The findings presented in this section put into evidence that a single artifact, the tagout tag (Figure 2), may both fulfill formal coordination functions and support ad hoc coordinative practices.

\subsubsection{Tags in formal coordination: their role}

Every energy isolating device that is part of an isolation boundary is padlocked and a tag is attached to it. The tag has red lettering: 'Locked. Do not manipulate.' ("Condamné. Ne pas manouvrer." on Figure 2). It objectifies the requirement for workers not to manipulate equipments that are in a tagout and can thus be thought of as a 'coordination mechanism', a formal coordination protocol imprinted upon an artifact.

The formal yet actual coordinative role performed by tags is very straightforward: it is the management of a shared resources (equipments) dependency. An equipment without a tag might be manipulated by practitioners from different departments while a tagged one requires prior authorization from the tagout supervisor to be manipulated.

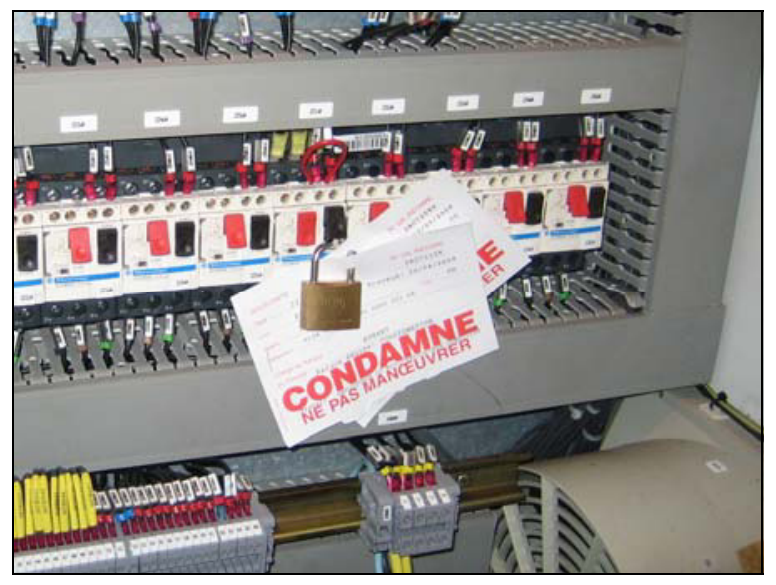

Fig. 2. A tag attached to a padlocked energy isolating device.

\subsubsection{Tags in coordinative practices: their use in accordance with professional culture}

When a maintenance activity is over, the corresponding tagout certificate has to be given back to the tagout supervisor. Then, a field operator (Operations department) unlocks the energy isolating devices in accordance with the tag hang sheet the tag supervisor provided her/him with.

A previous study [5] revealed that all field operators, in addition with complying with the requirement to write a checkmark on the tag hang sheet after removing a tag, keep with them the tags they remove. They then hand them to the tagout supervisor, alongside the marked tag hang sheet. The count of tags, which is fast and simple, provides the tagout supervisor with an extra mean to make sure the field operator took care of each and every tag she/he had to remove. If a tag is missing, comparing removed tags to the tag hang sheet is an easy way to identify the energy isolating device that remained padlocked.

This practice, although not prescribed by formal coordination, is widely used and belongs to the professional culture of tagout supervisors and field operators. It can be thought of a coordinative practice since it facilitates the management of the dependency that characterizes the relationship between the field operator and the tagout supervisor, when removing a tagout is concerned (the tags removal by the field operator is a pre-requisite for the tagout removal by the tagout supervisor). 


\subsubsection{Tags in coordinative practices: an innovative use}

This section presents a case (cf. 'Methods' section) made shorter by only keeping elements related to tags.

On a Wednesday morning, a maintenance work manager comes to the office of the tagout supervisor to have a tagout certificate delivered. The tagout supervisor gives it to him for signing. At this moment, the tagout supervisor phone rings. Since he knows it will take some time for the work manager to check the certificate before signing it, he answers the call.

After he hangs up the phone, the work manager tells him that there is a tag stapled to one of the sheets of the certificate. The tagout supervisor perfectly knows that tags are usually located on field, attached to isolation devices. He is thus quite surprised by the information the work manager just gave him. This element of surprise causes the tagout supervisor to focus his attention upon the stapled tag. His concern switches from 'delivering the certificate' to 'understanding why is a tag stapled to the certificate'.

He looks at the tag and deduces, from the tagout serial number it bears, that it is an Operations tagout tag. From the serial number and designation of the device the tag is associated to, he gets to know it concerns a valve that is usually locked in open position for the needs of Operations. He checks the tagout certificate and sees the valve was closed to secure the maintenance activity the work manager is about to carry out. With this short analysis, he understands that it is normal for the Operations tag not to be attached to the valve. Even if he cannot yet understand why the tag is stapled to the certificate, the certificate being printed and ready for delivery proves that the isolation boundary is formed. He then delivers the certificate to the work manager.

After the work manager has left, the tagout supervisor tries again to figure out the reason why the tag was stapled to the certificate. After a very short while, he infers that the tagout supervisor who had the Operations tag removed has been willing to draw attention to the fact the valve was to be closed, and the Operations tag consequently attached back, after the maintenance activity.

Should this inference be right (collected data does not allow to give a firm opinion), this case would reveal yet another possible use of tags for coordinative purposes. The surprise expressed by the tagout supervisor, however, shows that this hypothetical coordinative practice is not yet shared among the tagout supervisors community.

\section{Implications for design and future work}

The work done to date within the context of this study must be taken further to allow implications for the redesign of the tagout process to be enunciated. Nonetheless, this paper provides an example of the value of studying work practices 'in the wild' when it comes to contributing to innovation projects from an ergonomic perspective.

Indeed, as the presented findings show, fieldwork allows the identification of limitations in the tools (in the general sense of the word) practitioners are provided with by the organization. Shedding the light on such limitations can give new insights into what should been designed to support both the efficiency and safety of workers and organizations. Beyond marginal practices, practices that partake from a professional culture are most useful to discover. Drawing inspiration from them might improve appropriation of novelties.

The preliminary study presented in this paper is part of a broader research project that aims at informing the question of whether North American work organization regarding tagouts and its supporting tools could be of interest for redesigning EDF ones. With this respect, the next steps in the intended methodological approach are:

- Building a library of key situations regarding tagout practices in EDF plants (some situations reported in this paper will feed this library) and finding a way to model them;

- Identifying features of tagout practices which are specific to American utilities; Fieldwork is about to be carried out in the US;

- Assessing the potential impact of transferring the identified American features on the selected key situations; this will require defining an adequate method.

\section{References}

[1] J.E. Bardram and C. Bossen, A web of coordinative artifacts: collaborative work at a hospital ward, in: GROUP'05: Proceedings of the ACM Conference on Supporting Group Work, M. Pendergast, K. Schmidt, G. Mark and M. Ackerman, eds., ACM, New York, pp. 168-176.

[2] T.H. Benchekroun, B. Pavard and P. Salembier, Design of cooperative systems in complex dynamic environments, in: Expertise and technology: cognition and human-computer cooperation, J.-M. Hoc, C. Cacciabue and E. Hollnagell, eds., LEA, New Jersey, 1994, pp. 167-182. 
[3] C.A. Bolstad, H.M. Cuevas, C. Gonzalez and M. Schneider, Modeling shared situation awareness, Paper presented at the 14th Conference on Behavior Representation In Modeling and Simulation (BRIMS), Los Angeles, May 16-19th, 2005.

[4] H. Clark, Using language, Cambridge University Press, Cambridge, 1996.

[5] G. Filippi and F. Palaci, Analyzing work practices for design: the upgrading of field operators' computer devices, Paper presented at the 35th Enlarged Halden Programme Group Meeting, Storefjell, Norway, March 14-19, 2010.

[6] C. Heath and P. Luff, Collaboration and control: crisis management and multimedia technology in London underground line control rooms. Computer Supported Cooperative Work 1 (1992), 24-48.

[7] B. Johansson and E. Hollnagel, Pre-requisites for large scale coordination, Cognition, Technology and Work 9 (2007), 513.

[8] G. Klein, P.J. Feltovich, J.M. Bradshaw and D.D. Woods, Common ground and coordination in joint activity, in: Organizational simulation, W.B. Rouse and K.R. Boff, eds., John Wiley, New York, 2004, pp. 139-184.

[9] T.W. Malone and K. Crowston, The interdisciplinary study of coordination, ACM Computing Surveys 26 (1994), 87119.
[10]R. Randell, S. Wilson, P. Woodward and J. Galliers, Beyond handover: supporting awareness for continuous coverage, Cognition, Technology and Work 12 (2010), 271-283.

[11]L. Rognin, P. Salembier and M. Zouinar, Cooperation, reliability of socio-technical systems and allocation of function, International Journal of Human-Computer Studies 52 (2000), 357-379.

[12]K. Schmidt, Modes and mechanisms of interaction in cooperative work (Research report Risø-R-666(EN)), Risø National Laboratory, Denmark, 1994.

[13]K. Schmidt, Of maps and scripts: the status of formal constructs in cooperative work, in: GROUP '97: Proceedings of the international ACM SIGGROUP conference on Supporting group work, 1997, pp. 138-147.

[14]K. Schmidt and C. Simone, Coordination mechanisms: towards a conceptual foundation of CSCW systems design, Computer Supported Cooperative Work, 5 (1996), 155-200.

[15]J. Theureau, G. Filippi, G. Saliou, B. Le Guilcher and P. Vermersch, Cultural issues of nuclear power plant collective control in accidental situations and their impact upon design issues, Paper presented at ECCE-11, the 11th European Conference on Cognitive Ergonomics, Catania, Italy, September 8-11, 2002. 